\title{
The Application Research of Computer Database Technology in Information Management
}

\author{
HAN Qiang ${ }^{1, a}$ \\ ${ }^{1}$ Education Technology and Network Center, Jilin Teachers Institute of Engineering and \\ Technology, Changchun Jilin 130052, China \\ ahanqingjl@126.com
}

Keywords: Database Technology, Information Management, SQL Server

\begin{abstract}
With the rapid growth of domestic economy, science and technology level unceasing enhancement, the rapid development of electronic information technology, the computer database technology is widely used in various fields, playing an increasingly important role. Computer database technology in the application of information management, greatly improving the efficiency of information management, met the requirement of the complexity of the information management. However, before such a convenient and efficient technology, some domestic enterprises and institutions will appear stretched gaffes, therefore, the focus on the development of electronic information technology at the same time, more should focus on the cultivation of the talent, improve staff quality, so as to achieve mastering many skills while specializing in professionalism, and combined with computer database technology, to realize information management of high efficiency and rate of the query.
\end{abstract}

\section{Introduction}

The development of modern society, and the application of network technology in people's life is very extensive, companies are using the network information technology for data collection management, computer information base in the country's economic and cultural industries have great application, has brought great convenience to people's life [1]. Computer information management is the main way of data management, so the computer database technology is very important to people in the application of information management, only to strengthen the management of the application of the computer database technology, can increase work efficiency of people in the life of information management, information management become more accurate.

Database and information management system are complementary to each other, are inseparable whole, the combination of the two is the basis of the information construction and the safeguard, also is the important power to promote the development of science and technology [2]. With the deepening of the database and computer information management application, we also found some problems to be solved, such as database security protection needs to be improved, the updating of the database theory needs further in practice to prove, our ongoing research is needed to promote better and faster development of database technology, promote the information management better, more secure applications. Database technology in information management application flexibility and to promote the efficiency of use, will promote its to the larger data capacity and intelligent direction. As the technology level of ascension and the rapid expansion of the scope of application, the database technology in the user information management cost and the integration of database engine performance will be further improved.

\section{Application of database technology in the information management}

\section{The application of database in enterprise management}

With the development of network technology, network communication technology plays an important role in enterprise management. Large enterprises use database technology and network communication technology, the scattered around the branch link effectively, guarantee the company as a whole, from the aspects of global management [3]. At the same time, the company want to 
development, should be carried out as a whole and efficient and orderly. All kinds of information communication in the company may also influence the survival and development of the enterprise. The company size, the greater the need will be the more communication, if a single only rely on information communication between people, even in guarantee under the premise of no error, the efficiency of its limited inevitably influences the benefit of the company as a whole. However, if the use of database technology to process the information, to ensure the validity of the information already so, also greatly improve the processing efficiency [4].

The company's document management system, for example, is the use of database technology to manage the company's information. At the same time, the database technology in the construction of hospital information industry plays an important role. Hospital as a large business unit also needs the employees' attendance work, so the hospital also have staff attendance system etc., in order to save the patient's medical records and hospital and so on, gradually from paper files to the database file. On the basis of the database file more help doctors check, at the same time can also help the patient after the transfer of the communication between cases. Database technology can be applied not only in the enterprise information management, also can be used in schools and other institutions of information management, such as IC card system of the students from the school.

\section{The application of database in the product}

In nowadays, more and more products, some products not only need to record a large number of monitoring data, at the same time also need certain processing these data, in order to effectively management. Some need long time monitoring equipment, for example, its measuring the amount of data is quite large, using database technology awards these data storage and processing according to certain rules, so that it can be efficient analysis of monitoring data. At the same time, some software products, such as network game, etc., need to establish a more complete database so that the player's account and password for storage and management, etc.

\section{Key technology of computer database}

As an important part of modern information science and technology, database technology is the core of computer data processing and information management system. It how to research and solve in the process of computer information processing effectively organize large amounts of data, and data storage, reduce data storage redundancy in the database system, realize the sharing of data, ensure the safety of the data and to quickly retrieve and process data, and so on [5]. Data is the database technology research and management of objects, that would limit the database technology involved in the specific contents should include: the specified structure to establish the corresponding database and data warehouse can be through the unification of the data organization and management to achieve; In database management system and the data mining system for the design, should realize the user to add, modify, delete data in the database, processing, analysis, understanding, reporting and printing and so on a variety of functions; Using application management system finally realizes to the data processing, analysis and understanding.

SQL Server is a large and complex software packages. It is the core of a powerful relational data engine, but also contains the related services, tools and development technology, the analysis of the main service component is shown in figure 1.

The database Engine is the core of the entire database management system, SQL Server database Engine itself consists of two main components: the Query Processor (Query Processor) and Storage Engine (Storage Engine). Query processor use SQL statements to express, it SQL statements can be divided into a number of atomic other steps, the query can be serial or parallel processing. Furthermore, it will choose a query plan in a certain range, and the appropriate use of the index. Storage engine's mission is to be responsible for the operation of database on the physical structure, organization structure of the database file, it directly access the data on the disk to complete the corresponding function. 


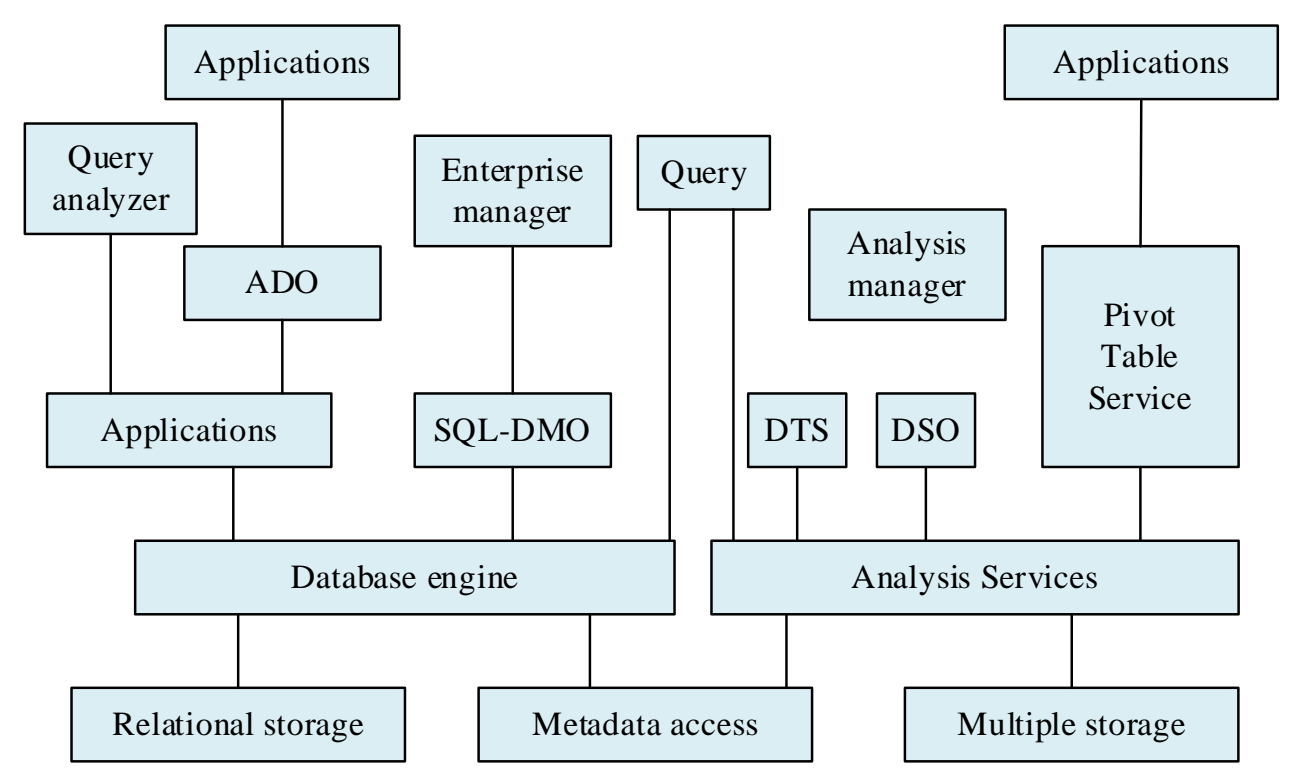

Figure 1.Service component of computer database

\section{Research of SQL database model}

Database system is the core and basis of the data type, it is also the real world in the database abstraction. Data model generally includes three elements, namely, data structure, data operation and data integrity constraints. Data structure is mainly used to describe the static characteristics of the data, the relationship between the data structure and data; Data manipulation is to point to to the existing data in the database query, modify, delete, or add new data of various data access method, also may include data access relevant rules; Data integrity constraints, composed of a set of integrity rules. SQL (Structured Query Language) is a kind of between relational algebra and relational calculus of Structured Query Language (SQL), its function is not only a Query. SQL is a universal language of relational database, function is extremely strong. SQL statement data can be obtained from a relational database, can also use SQL statements to create the database, modify the data, and increasing data, and so on. SQL language is widely used, mainly has the characteristics such as easy to use, easy to learn, and as an industry standard language database operation. Such as DB2, ORACLE relational database products have realized the SQL language. Support relational database SQL language level 3 model structure, as shown in figure 2. Contact the model view, part of the base table, contact the base table model, model within contact store file.

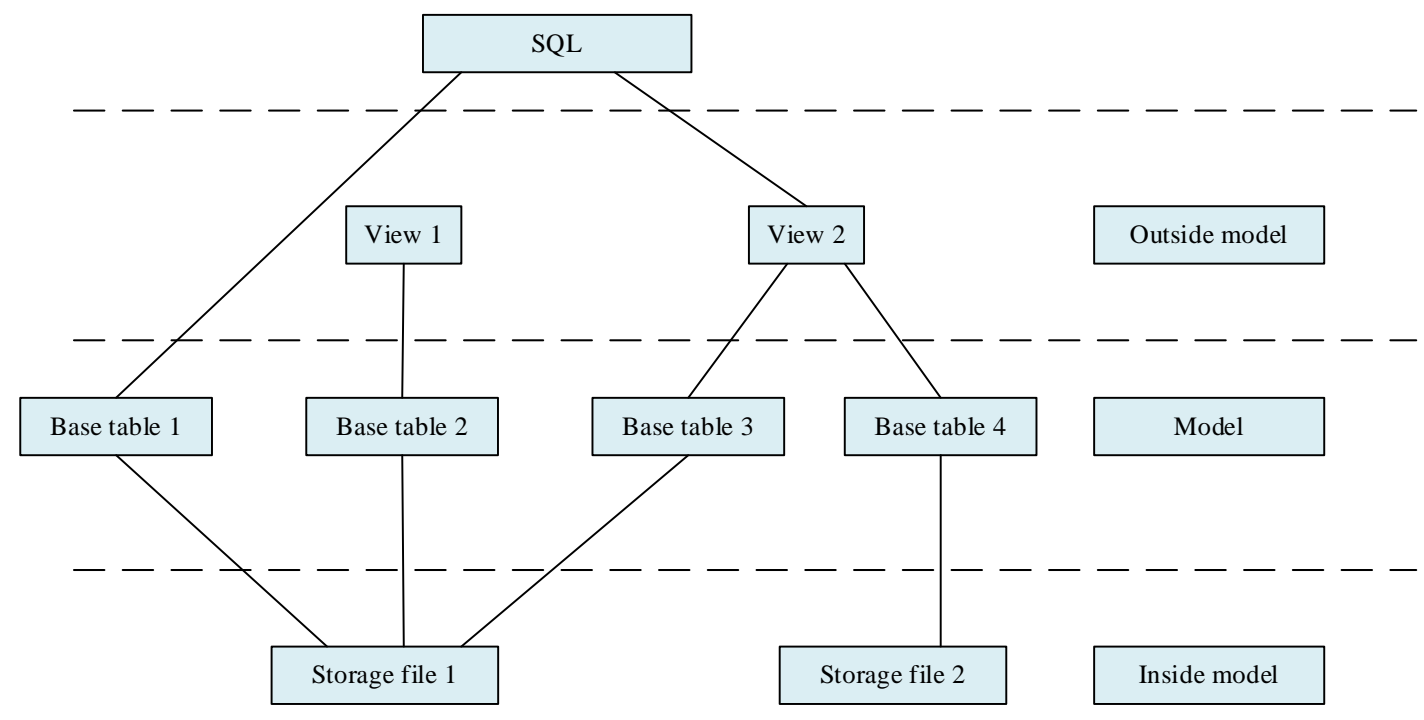

Figure 2. SQL relational database model structure 
Users for the base table, view, query or related operations can be done by writing SQL language, the base table and view is consistent, in SQL said. The base table in SQL is corresponds to a relationship, itself is the independent existence. One or more basic table corresponds to a storage file, a table can have several indexes, and storage file also contains these indexes. Model in the relational database is composed of the logical structure of storing files, and the physical structure of the storage file is transparent to users, and is arbitrary. View itself is not stored in the database, it derived from one or a few basic table. Only views are stored in the database has a characteristic is, corresponding to the definition, but does not store view the corresponding data, the database export basic deposit corresponding data in the table view, can be thought of as essentially a view is a virtual table. From the aspects of concept, the view and the base table is consistent, the user in the view on the basis of defining a view again.

\section{Conclusion}

Computer database good applicability and determines the future good development trend of modernity, improve on the technical level and information management technology constantly improve the momentum of development, the database technology will be caught the attention of the height of the social development in various fields. But in the computer database system is a good development trend, we should still keep on system builds a clear cognition, database technology are still many shortcomings in the information management, this needs us to continuously explore and innovation in the development of database technology, in order to realize the database technology, more efficient, more secure applications.

\section{References}

[1] A.S. Voulodimos, C.Z. Patrikakis, A.B. Sideridis: Computers and Electronics in Agriculture, Vol.70(2010) No.2, p. 380.

[2] N.A. Sultan: International journal of information management, Vol.31 (2011) No.3, p. 272.

[3] S. Chaudhuri, U. Dayal, and V. Narasayya: Communications of the ACM, Vol.54 (2011) No.8, p. 88.

[4] N. Leavitt : Computer, Vol.43 (2010) No.2, p. 12.

[5] N. Sultan: International Journal of Information Management, Vol.30 (2010) No.2, p.109. 2010, 30(2): 109-116. 